\title{
Raman Spectroscopy for the Endoscopic Diagnosis of Esophageal, Gastric, and Colonic Diseases
}

\author{
Neel Sharma, Nobuyoshi Takeshita and Khek Yu Ho \\ Division of Gastroenterology and Hepatology, National University Health System, Singapore
}

Globally white-light endoscopy with biopsy sampling is the gold standard diagnostic modality for esophageal, gastric, and colonic pathologies. However, there is overwhelming evidence to highlight the deficiencies of an approach based predominantly on eyeball visualization. Biopsy sampling is also problematic due in part to excessive sampling and hence attendant cost. Various innovations are currently taking place in the endoscopic domain to aid operators in diagnosis forming. These include narrow band imaging which aims to enhance the surface anatomy and vasculature, and confocal laser endomicroscopy which provides real time histological information. However, both of these tools are limited by the skill of the operator and the extensive learning curve associated with their use. There is a gap therefore for a new form of technology that relies solely on an objective measure of disease and reduces the need for biopsy sampling. Raman spectroscopy (RS) is a potential platform that aims to satisfy these criteria. It enables a fingerprint capture of tissue in relation to the protein, DNA, and lipid content. This focused review highlights the strong potential for the use of RS during endoscopic gastroenterological examination. Clin Endosc 2016;49:404-407

Key Words: Spectrum analysis, Raman; Real-time diagnosis; Real-time decision making; Narrow band imaging; Confocal endomicroscopy

\section{INTRODUCTION}

White-light endoscopy with biopsy sampling has been established globally as the gold standard diagnostic modality for esophageal, gastric, and colonic pathologies. However, overwhelming evidence highlights the deficiencies of approaches based predominantly on eyeball visualization. Biopsy sampling is also problematic partly because of excessive sampling and hence attendant cost. Various innovations in the endoscopic domain are currently underway to aid operators in diagnosis. These include narrow band imaging, which is aimed at enhancing the surface anatomy and vasculature, and

Received: July 14, 2016 Revised: August 23, 2016

Accepted: August 24, 2016

Correspondence: Khek Yu Ho

Division of Gastroenterology and Hepatology, National University Health System (NUHS), Tower Block Level 10, 1E Kent Ridge Road, 119228, Singapore

Tel: +65-6772-4362, E-mail: khek_yu_ho@nuhs.edu.sg

(cc) This is an Open Access article distributed under the terms of the Creative Commons Attribution Non-Commercial License (http://creativecommons.org/ licenses/by-nc/3.0) which permits unrestricted non-commercial use, distribution, and reproduction in any medium, provided the original work is properly cited. confocal laser endomicroscopy, which provides real-time histological information. However, both of these tools are limited by the skill of the operator and the extensive learning curve associated with their use (Table 1).

Therefore, a new form of technology that relies solely on an objective diagnostic parameter of disease and reduces the need for biopsy sampling needs to be developed. Raman spectroscopy (RS) is a potential platform that aims to satisfy these criteria. In brief, RS involves the application of light that induces molecular polarization changes and inelastic scattering of incident photons that correspond to the exact nature of the Raman vibrational modes of the molecules in question. ${ }^{1}$ It enables fingerprint (FP) capture of tissue in relation to the protein, DNA, and lipid contents.

This focused review highlights the strong potential for the use of RS during endoscopic gastroenterological examinations.

\section{ESOPHAGUS}

Wang et al. ${ }^{2}$ used surface-enhanced Raman scattering (SERS) 
Table 1. Summary of Benefits and Drawbacks of the Currently Used Endoscopic Tools

\begin{tabular}{ll}
\hline Endoscopic tool & \multicolumn{1}{c}{ Benefits and drawbacks } \\
\hline White-light endoscopy & Requires little training but has poor sensitivity for lesion detection \\
Narrow band imaging & Shows enhanced surface anatomy and vasculature but has complicated classification systems \\
Confocal laser endomicroscopy & Provides real-time histological details but has a steep learning curve \\
Autofluorescence imaging & $\begin{array}{l}\text { Poor sensitivity and specificity in lesion detection } \\
\text { Chromoendoscopy }\end{array}$ \\
$\begin{array}{l}\text { Capable of enhanced surface lesion detection but has a strong interobserver disagreement } \\
\text { Raman spectroscopy }\end{array}$ & $\begin{array}{l}\text { Useful for objective diagnosis and requires little training. Its current drawbacks include its } \\
\text { ability to only diagnose focal lesions. }\end{array}$ \\
\hline
\end{tabular}

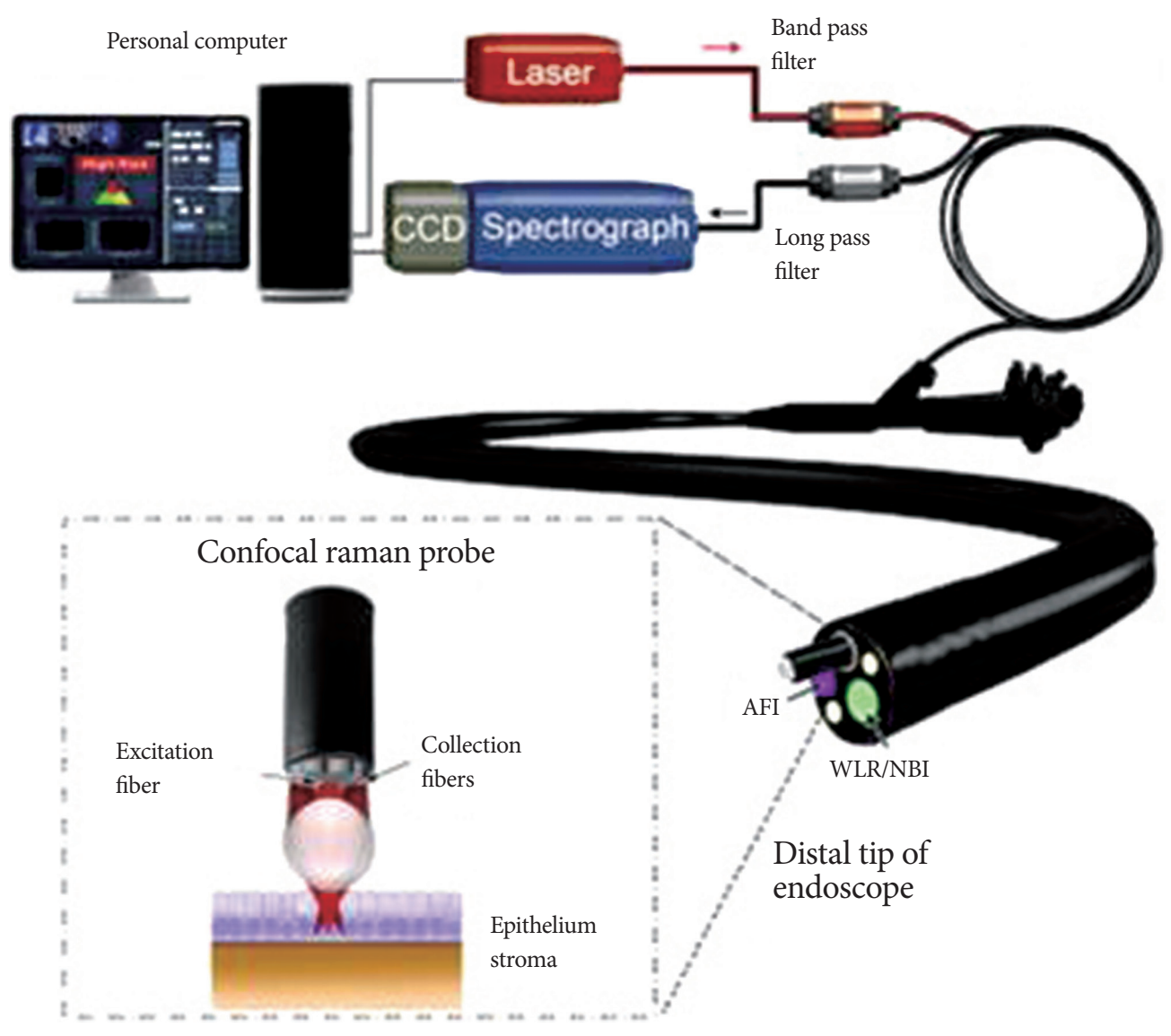

Fig. 1. Schematic diagram of the custom-built in vivo Raman spectroscopy system at National University Health System. Adapted from Bergholt et al., ${ }^{1}$ with permission from Elsevier. CCD, charge coupled device; AFI, autofluorescence imaging; WLR, white-light reflectance; NBI, narrow band imaging.

nanoparticles (NPs) to aid in the detection of cell surface biomarkers specific to esophageal cancer. A validated spectral endoscope was developed to aid in imaging SERS NPs that target the epidermal growth factor receptor and human epidermal growth factor receptor 2 by applying them in a rat esophageal cancer model. Whole luminal scanning was achieved at a speed of $0.6 \mathrm{~cm} / \mathrm{min}$. The highlighted advantages of SERS NPs are their excitation ability at a single wavelength $(785 \mathrm{~nm})$, enabling equal analysis of all NP reporters in terms of intensity, area, and depth. ${ }^{2}$
Bergholt and colleagues ${ }^{1}$ at the National University Hospital Singapore developed an in vivo Raman spectroscopic system for the real-time detection of Barrett's associated dysplasia (Fig. 1). The custom-built probe was designed in such a way as to enable its insertion within the preexisting endoscopic biopsy channel with subsequent placement on surface lesions. Results obtained noted a sensitivity and specificity of $87 \%$ and $84.7 \%$, respectively, for high-grade dysplastic tissue. ${ }^{1}$

Further work in relation to Barrett's associated dysplasia and adenocarcinoma conducted an ex vivo analysis of 62 patients. 
A sensitivity of $86 \%$ and specificity of $88 \%$ for the detection of both tissue subtypes were reported. ${ }^{3}$

Previous work by the same group involved measuring 337 Raman spectra from esophageal tissue samples from $28 \mathrm{pa}-$ tients. Analysis to distinguish between Barrett's esophagus and neoplasia demonstrated a sensitivity of $88 \%$ and a specificity of $87 \%$ for malignant disease. ${ }^{4}$

In relation to esophageal cancer, RS was used to assess 75 esophageal tissue samples from 27 patients. Esophageal cancer tissue demonstrated Raman signals associated with several aspects, including cell proliferation, decrease in lipid level, dysfunctional nuclear activity, and neovascularization. Elements such as actin, DNA, histones, triolein, and glycogen yielded an accuracy of $96.0 \%$ (i.e., sensitivity of $97.0 \%$ and specificity of $95.2 \%)$ for an in vivo diagnosis of disease. ${ }^{5}$

\section{STOMACH}

Wang et al. ${ }^{6}$ used RS to assess premalignant gastric pathologies. They obtained 5,792 Raman spectra from 441 tissue sites in 191 gastric patients. Diagnostic sensitivity rates of $96.0 \%$, $81.8 \%$, and $88.2 \%$, and specificity rates of $86.7 \%, 95.3 \%$, and $95.6 \%$ were obtained for the classification of normal, dysplastic, and cancerous gastric tissues, respectively. ${ }^{6}$

The same group used RS in the assessment of gastric intestinal metaplasia (IM). They obtained 4,520 Raman spectra from 157 patients. Diagnostic sensitivity rates of $89.3 \%, 89.3 \%$, and $75.0 \%$; specificity rates of $92.2 \%, 84.4 \%$, and $82.0 \%$; positive predictive values of $52.1 \%, 35.2 \%$, and $28.4 \%$; and negative predictive values of $98.9 \%, 98.8 \%$, and $97.2 \%$ were achieved by using a combined FP/high wave number (HW), FP, and HW Raman process, respectively. Further analysis demonstrated an area under the curve of 0.92 for IM. ${ }^{7}$

Another group successfully used RS for endoscopic assessment of premalignant lesions (adenomatous polyp, $n=27$ ) and cancer tissues (adenocarcinoma, $n=33$ ). Sensitivity rates of $96.3 \%, 96.9 \%$, and $96.9 \%$, respectively, and specificity rates of $93 \%, 100 \%$, and $95.2 \%$, respectively, were observed in discrimination of normal, adenomatous polyp, and adenocarcinoma gastric tissues. $^{8}$

Bergholt et al. ${ }^{9}$ used RS in the detection of precancerous gastric IM and dysplasia. In an analysis of 1,277 spectra from a patient sample of 83 , results indicated that RS is useful for identifying normal mucosa at a sensitivity of $75.88 \%$ and specificity of $87.21 \%$, IM at a sensitivity of $46.67 \%$ and specificity of $87.55 \%$, dysplasia at a sensitivity of $83.33 \%$ and specificity of $95.80 \%$, and adenocarcinoma at a sensitivity of $84.91 \%$ and specificity of $95.57 \%{ }^{9}$

Additional gastric work comes from a total of 2,748 gastric tissue spectra, encompassing a patient sample size of 305. RS provided a diagnostic accuracy of $85.6 \%$, sensitivity of $80.5 \%$, and specificity of $86.2 \%$ for the detection of gastric cancer. ${ }^{10}$

Kawabata et al. ${ }^{11}$ obtained 213 Raman spectra from 12 malignant gastric lesions. A sensitivity, a specificity, and an accuracy of $73 \%, 73 \%$, and $72 \%$, respectively, were attained for cancer diagnosis. RS proved valuable in the differentiation between tumor grades and between early and advanced cancers, with a focus on Tla (M) and T1b (SM) with high accuracy (98\%, 93\%, and $98 \%$, respectively). ${ }^{11}$

The molecular capabilities of RS can help to determine the biological changes that occur from normal to malignant tissues. This provides added value in determining potential cell surface markers suitable for enhanced endoscopic diagnosis and possible future drug targets. Chen et al. ${ }^{12}$ used RS to analyze the biochemical nature of molecules associated with malignant gastric mucosa. Results demonstrated a notable DNA phosphate backbone instability in cancer cells with an increase in the relative content of histones; a reduction in collagen content, enhancing metastasis; and an increase in unsaturated fatty acid levels. ${ }^{12}$

\section{COLON}

Work in relation to the real-time diagnosis of adenomatous polyps has been undertaken by using RS. The FP/HW Raman spectra of 17 colorectal polyps from 50 patients were obtained. Raman spectral differences between hyperplastic lesions and adenomas that were significant at $p<0.001$ were observed. This was speculated to be due to changes in protein, DNA, and lipid contents. Simultaneous FP/HW RS provided a diagnostic sensitivity of $90.9 \%$ and specificity of $83.3 \%$ for differentiating adenomas from hyperplastic polyps. ${ }^{13}$

Additional research by the same group used RS for several anatomical colonic sites from the rectosigmoid through the ascending colon in 50 patients. In this regard, a diagnostic accuracy of $88.8 \%$ (sensitivity $93.9 \%$ and specificity $88.3 \%$ ) was reported for colorectal cancer detection. ${ }^{14}$

Pre-malignant stages of colorectal cancer can also benefit from the use of RS. Veenstra et al. ${ }^{15}$ evaluated the spectra of patients with ulcerative colitis, focusing on the mucosal and serosal regions. Regarding the mucosa, RS had a sensitivity of $82 \%$ and specificity of $89 \%$. For the serosal regions, RS had a sensitivity of $87 \%$ and a specificity of $93 \%{ }^{15}$

\section{CONCLUSIONS}

Limitations of endoscopic examination are well known. 
The inability of white-light endoscopy to truly distinguish malignant lesions from inflammatory lesions is a persistent issue, and only astute examination can help reduce missed lesions. While additional modalities such as narrow band imaging aim to enhance the surface anatomy of lesions, classification systems are complex and require extensive training. RS is aimed at providing a more objective endoscopic diagnosis. Thus, it provides several benefits, including limited or a more targeted biopsy sampling. In view of the current guidelines established by the American Society for Gastrointestinal Endoscopy for Preservation and Incorporation of Valuable Endoscopic Innovations, which focus on real-time diagnosis and treatment, RS allows for real-time diagnosis and further aids endoscopic resection through appropriate margin detection. Currently, pathological examination techniques remain the gold standard diagnostic method. Further research will allow RS to provide clinicians with significant advantage in the detection of malignant and pre-cancerous pathologies.

Conflicts of Interest

K.Y.H. is cofounder of EndoFotonics Pte Ltd.

\section{Acknowledgments}

NS would like to acknowledge Mads Bergholt, Lin Kan, Jianfeng Wang, and Zhiwei Huang for their mentorship on RS during his clinical research fellowship.

\section{REFERENCES}

1. Bergholt MS, Zheng W, Ho KY, et al. Fiberoptic confocal raman spectroscopy for real-time in vivo diagnosis of dysplasia in Barrett's esophagus. Gastroenterology 2014;146:27-32.

2. Wang YW, Kang S, Khan A, Bao PQ, Liu JT. In vivo multiplexed molec- ular imaging of esophageal cancer via spectral endoscopy of topically applied SERS nanoparticles. Biomed Opt Express 2015;6:3714-3723.

3. Almond LM, Hutchings J, Lloyd G, et al. Endoscopic Raman spectroscopy enables objective diagnosis of dysplasia in Barrett's esophagus. Gastrointest Endosc 2014;79:37-45.

4. Almond LM, Hutchings J, Kendall C, et al. Assessment of a custom-built Raman spectroscopic probe for diagnosis of early oesophageal neoplasia. J Biomed Opt 2012;17:081421-081421.

5. Bergholt MS, Zheng W, Lin K, et al. In vivo diagnosis of esophageal cancer using image-guided Raman endoscopy and biomolecular modeling. Technol Cancer Res Treat 2011;10:103-112.

6. Wang J, Lin K, Zheng W, et al. Fiber-optic Raman spectroscopy for in vivo diagnosis of gastric dysplasia. Faraday Discuss 2016;187:377-392.

7. Lin K, Wang J, Zheng W, et al. Rapid fiber-optic Raman spectroscopy for real-time in vivo detection of gastric intestinal metaplasia during clinical gastroscopy. Cancer Prev Res (Phila) 2016;9:476-483.

8. Luo S, Chen C, Mao H, Jin S. Discrimination of premalignant lesions and cancer tissues from normal gastric tissues using Raman spectroscopy. J Biomed Opt 2013;18:067004.

9. Bergholt MS, Zheng W, Ho KY, et al. Fiber-optic Raman spectroscopy probes gastric carcinogenesis in vivo at endoscopy. J Biophotonics 2013;6:49-59.

10. Duraipandian S, Sylvest Bergholt M, Zheng W, et al. Real-time Raman spectroscopy for in vivo, online gastric cancer diagnosis during clinical endoscopic examination. J Biomed Opt 2012;17:081418.

11. Kawabata T, Kikuchi H, Okazaki S, et al. Near-infrared multichannel Raman spectroscopy with a $1064 \mathrm{~nm}$ excitation wavelength for ex vivo diagnosis of gastric cancer. J Surg Res 2011;169:e137-e143.

12. Chen Y, Dai J, Zhou X, Liu Y, Zhang W, Peng G. Raman spectroscopy analysis of the biochemical characteristics of molecules associated with the malignant transformation of gastric mucosa. PLoS One 2014;9:e93906.

13. Bergholt MS, Lin K, Wang J, et al. Simultaneous fingerprint and highwavenumber fiber-optic Raman spectroscopy enhances real-time in vivo diagnosis of adenomatous polyps during colonoscopy. J Biophotonics 2016;9:333-342

14. Bergholt MS, Zheng W, Lin K, et al. Characterizing variability of in vivo Raman spectroscopic properties of different anatomical sites of normal colorectal tissue towards cancer diagnosis at colonoscopy. Anal Chem 2015;87:960-966.

15. Veenstra MA, Palyvoda $\mathrm{O}$, Alahwal $\mathrm{H}$, et al. Raman spectroscopy in the diagnosis of ulcerative colitis. Eur J Pediatr Surg 2015;25:56-59. 